\title{
Risk stratification and therapeutics of neuroblastoma: the challenges remain
}

\author{
Hong Mei, Zhen-Yu Lin, Qiang-Song Tong \\ Wuhan, China
}

$\mathrm{P}$ ediatric oncology is a rapidly evolving and exciting field. Although advances have been made in this field through the integration of systematic basic laboratory and clinical research, treatment outcomes are still suboptimal for patients with a malignant solid tumor. Neuroblastoma, the most common extracranial solid tumor of childhood, accounts for $7 \%-8 \%$ of all cancers in children in the United States and United Kingdom. ${ }^{[1,2]}$ It typically originates from the adrenal gland, sympathetic ganglia, paraganglia, or along the sympathetic nervous system, and can undergo metastasis to the liver, bones, brain, and skin. ${ }^{[3]}$ The tumorigenesis of neuroblastoma involves both embryonic and oncogenic factors, thus, its biological and clinical features are highly heterogeneous. ${ }^{[4]}$ The heterogeneous nature of this disease is best exemplified by its contrasting clinical outcomes among different groups of patients. Some neuroblastomas can spontaneously regress or mature into benign ganglioneuroma. Some of these neuroblastoma patients follow a watch-andwait approach as the first-line therapy in all current treatment protocols. ${ }^{[5]}$ And for early-staged (stages 1 and 2) patients, they are often cured by surgery alone, without the adjuvant use of chemotherapy or radiotherapy ${ }^{[6]}$ However, a large proportion of patients present rapid progression and poor prognosis, despite multimodal treatment strategies, such as surgery, chemoradiotherapy, hematopoietic stem cell transplantation, and anti-disialoganglioside

Author Affiliations: Department of Pediatric Surgery, Union Hospital, Tongji Medical College, Huazhong University of Science and Technology, Wuhan, China (Mei H, Tong QS); Cancer Center, Union Hospital, Tongji Medical College, Huazhong University of Science and Technology, Wuhan, China (Lin ZY)

Corresponding Author: Qiang-Song Tong, PhD, MD, Department of Pediatric Surgery, Union Hospital, Tongji Medical College, Huazhong University of Science and Technology, Wuhan 430022, China (Tel: 86-2785726005; Email: qs_tong@hotmail.com)

doi: 10.1007/s12519-016-0001-6

(C)Children's Hospital, Zhejiang University School of Medicine, China and Springer-Verlag Berlin Heidelberg 2016. All rights reserved.

immunotherapy combined with cytokines. ${ }^{[7,8]}$ Thus, neuroblastoma has been one of the most fascinating and also frustrating diseases in pediatric oncology for several years.

In view of the biological heterogeneity and dissimilar therapeutic approaches of neuroblastoma, it is commonly agreed that accurate risk estimation at the time of diagnosis is a pivotal prerequisite for the clinical management of this disease ${ }^{[9]}$ Apart from a multitude of factors including the patient's age at diagnosis, tumor histopathology, and disease staging, the outcome of treatment also depends on molecular and genetic abnormalities. ${ }^{[9,10]}$ In the rare familial neuroblastoma cases (less than 2\%), the patients present with germline mutations of genes, such as paired-like homeobox $2 b,{ }^{[11]}$ anaplastic lymphoma receptor tyrosine kinase $(A L K),{ }^{[12]}$ and breast cancer 1-associated RING domain protein $1 .^{[13]}$ In the majority of patients with tumors of sporadic origin, the most commonly encountered molecular and genetic lesions portending a poorer prognosis include chromosome $1 \mathrm{p}$ deletion, gain of chromosome $17 \mathrm{q}$, somatic mutations or amplification of $A L K(9 \%$ $14 \%$ ), activating mutations of $\alpha$-thalassemia/mental retardation syndrome $\mathrm{X}$-linked, and amplification of $\mathrm{v}$-myc avian myelocytomatosis viral oncogene neuroblastoma derived homolog (MYCN) (20\%$30 \%){ }^{[14-16]}$ According to these prognostic factors, the patients are stratified into subgroups with expected low, intermediate and high risk of death from disease, and contemporary treatment of neuroblastoma is also varied.$^{[17]}$ However, we commonly encounter the confusing situation in clinic that patients with the same pattern of markers, while receiving the same treatment, may have highly contrasting clinical courses. The estimated 5-year event-free survival rate of highrisk NB is lower than $50 \%$, and its treatment remains challenging. Therefore, these risk estimation strategies are imperfect and non-individualized, and it is urgent to standardize criteria for evaluation of the disease and determine more optimal individual therapy for neuroblastoma.

In recent years, scientists enthusiastically embrace the impressive genomic information and molecular

World J Pediatr, Vol 12 No 1 · February 15, 2016 · www.wjpch.com 
fundamentals of this disease. With the development of next-generation sequencing (NGS) analyses, it is feasible to assess the genetic alterations of neuroblastoma in a genome-wide manner. ${ }^{[18]}$ Moreover, refined genetic analyses can disclose a subgroup of aggressive tumors that have distinct global alteration patterns and divergent outcomes. It is hopeful that these advances will eventually culminate in more individual and effective treatment for the children with neuroblastoma. Disappointingly, evaluation of global alteration pattern from NGS analyses reveals that only few amino acid-changing mutations contribute to the basis of neuroblastoma, which could not be incorporated into clinical diagnostic use. ${ }^{[13]}$ In the past few years, emerging evidence shows that a unique panel of microRNA (miRNA) is associated with the diagnosis and prognosis of neuroblastoma,${ }^{[19]}$ and some specific miRNAs are considered as independent prognosis factors in multivariable analysis, such as miR-17-92 $2^{[20]}$ and miR-21. ${ }^{[21]}$ The roles of miRNAs in individualized treatment of cancer patients are very promising, and the targets and potential cellular functions of these miRNAs implicated in neuroblastoma warrant further studies. In addition, recent evidence shows the crucial roles of long non-coding RNA (lncRNA) in the tumorigenesis and progression of neuroblastoma, such as non-coding RNA expressed in aggressive neuro-blastoma, ${ }^{[22]}$ neuroblastoma associated transcript- $1,{ }^{[23]}$ paired box 6 upstream antisense RNA, ${ }^{[24]}$ and MYCN opposite strand. ${ }^{[25]}$ High levels of a novel lncRNA, named as lncUSMycN, are independently associated with poor clinical outcome of neuroblastoma patients. ${ }^{[2]}$ Importantly, the combination analysis of different lncUSMycN cutoff values and well-established factors is useful for individual risk assessment in different cohorts of neuroblastoma patients. ${ }^{[26]}$ Therefore, IncRNAs are expected to have a significant impact on clinical oncology in the next decade, and might be potential novel targets for the treatment of neuroblastoma.

In summary, a pressing challenge in neuroblastoma is to standardize the risk stratification and improve the predictive accuracy based on individual patterns of genetic alternation, which will help us to optimize the individual and appropriately tailor therapy, and avoid both over- and under-treatment for patients. Although a number of molecular and genetic factors are discovered to indicate the stage and risk classification, there is still a lot of work to do for clinical application. It is considerably optimistic that the future treatment of neuroblastoma is likely to change dramatically in the coming years.

Funding: National Natural Science Foundation of China (81101905, 81272779, 81372667, 81372401, 81472363, 81402301,
81402408, 81572423), Fundamental Research Fund for the Central Universities (2012QN224, 2013ZHYX003, 01-18-530112, 01-18530115), and Natural Science Foundation of Hubei Province (2014CFA012).

Ethical approval: Not needed.

Competing interest: All authors disclosed no conflict of interest. Contributors: Mei H and Lin ZY wrote the initial draft. Tong QS reviewed and revised the manuscript. All authors approved the final version.

\section{References}

1 Maris JM. Recent advances in neuroblastoma. N Engl J Med 2010;362:2202-2211.

2 Finklestein JZ, Gilchrist GS. Recent advances in neuroblastoma. Calif Med 1972;116:27-36.

3 Moreno L, Marshall LV, Pearson AD. At the frontier of progress for paediatric oncology: the neuroblastoma paradigm. Br Med Bull 2013;108:173-188.

4 Kagedal B. Detecting minimal residual disease in neuroblastoma: still a ways to go. Clin Chem 2009;55:1268-1270.

5 Maris JM, Hogarty MD, Bagatell R, Cohn SL. Neuroblastoma. Lancet 2007;369:2106-2120.

6 Lee AC. Neuroblastoma: the challenge remains. Singapore Med J 2012;53:1-2.

7 Park JR, Eggert A, Caron H. Neuroblastoma: biology, prognosis, and treatment. Hematol Oncol Clin North Am 2010;24:65-86.

8 Park JR, Bagatell R, London WB, Maris JM, Cohn SL, Mattay KK, et al. Children's Oncology Group's 2013 blueprint for research: neuroblastoma. Pediatr Blood Cancer 2013;60:985993.

9 Oberthuer A. Genomic markers for neuroblastoma risk estimation: superseding tumor stage, age and MYCN? Biomark Med 2013;7:905-908

10 Bottino C, Dondero A, Bellora F, Moretta L, Locatelli F, Pistoia V, et al. Natural killer cells and neuroblastoma: tumor recognition, escape mechanisms, and possible novel immunotherapeutic approaches. Front Immunol 2014;5:56.

11 Bourdeaut F, Trochet D, Janoueix-Lerosey I, Ribeiro A, Deville A, $\mathrm{Coz} C$, et al. Germline mutations of the paired-like homeobox 2B (PHOX2B) gene in neuroblastoma. Cancer Lett 2005;228:5158 .

12 Bresler SC, Weiser DA, Huwe PJ, Park JH, Krytska K, Ryles H, et al. ALK mutations confer differential oncogenic activation and sensitivity to ALK inhibition therapy in neuroblastoma. Cancer Cell 2014;26:682-694.

13 Pugh TJ, Morozova O, Attiyeh EF, Asgharzadeh S, Wei JS, Auclair D, et al. The genetic landscape of high-risk neuroblastoma. Nat Genet 2013;45:279-284.

14 Cheung NK, Dyer MA. Neuroblastoma: developmental biology, cancer genomics and immunotherapy. Nat Rev Cancer 2013;13:397-411.

15 Pichler M, Calin GA. Long noncoding RNA in neuroblastoma: new light on the (old) N-Myc story. J Natl Cancer Inst 2014;106. doi: $10.1093 /$ jnci/dju150.

16 Tomioka N, Oba S, Ohira M, Misra A, Fridlyand J, Ishii S, et al. Novel risk stratification of patients with neuroblastoma by genomic signature, which is independent of molecular signature. Oncogene 2008;27:441-449.

17 Vermeulen J, De Preter K, Mestdagh P, Laureys G, Speleman 
F, Vandesompele J. Predicting outcomes for children with neuroblastoma. Discov Med 2010;10:29-36.

18 Molenaar JJ, Koster J, Zwijnenburg DA, van Sluis P, Valentijn LJ, van der Ploeg I, et al. Sequencing of neuroblastoma identifies chromothripsis and defects in neuritogenesis genes. Nature 2012;483:589-593.

19 Mei H, Lin ZY, Tong QS. The roles of microRNAs in neuroblastoma. World J Pediatr 2014;10:10-16.

20 Mestdagh P, Bostrom AK, Impens F, Fredlund E, Van Peer G, De Antonellis $\mathrm{P}$, et al. The miR-17-92 microRNA cluster regulates multiple components of the TGF-beta pathway in neuroblastoma. Mol Cell 2010;40:762-773.

21 Chen Y, Tsai YH, Fang Y, Tseng SH. Micro-RNA-21 regulates the sensitivity to cisplatin in human neuroblastoma cells. J Pediatr Surg 2012;47:1797-1805.

22 Yu M, Ohira M, Li Y, Niizuma H, Oo ML, Zhu Y, et al. High expression of ncRAN, a novel non-coding RNA mapped to chromosome $17 \mathrm{q} 25.1$, is associated with poor prognosis in neuroblastoma. Int J Oncol 2009;34:931-938.
23 Pandey GK, Mitra S, Subhash S, Hertwig F, Kanduri M, Mishra K, et al. The risk- associated long noncoding RNA NBAT-1 controls neuroblastoma progression by regulating cell proliferation and neuronal differentiation. Cancer Cell 2014;26:722-737.

24 Vance KW, Sansom SN, Lee S, Chalei V, Kong L, Cooper SE, et al. The long non-coding RNA Paupar regulates the expression of both local and distal genes. EMBO J 2014;33:296-311.

25 Zhao X, Li D, Pu J, Mei H, Yang D, Xiang X, et al. CTCF cooperates with noncoding RNA MYCNOS to promote neuroblastoma progression through facilitating $\mathrm{MYCN}$ expression. Oncogene 2015. doi: 10.1038/onc.2015.422.

26 Liu PY, Erriquez D, Marshall GM, Tee AE, Polly P, Wong M, et al. Effects of a novel long noncoding RNA, IncUSMycN, on $\mathrm{N}-\mathrm{Myc}$ expression and neuroblastoma progression. J Natl Cancer Inst 2014;106. doi: 10.1093/jnci/dju113.

Received December 10, 2015 Accepted after revision December 17, 2015 